\title{
BMJ Open Efficacy of bisphosphonates in specific knee osteoarthritis subpopulations: protocol for an OA Trial Bank systematic review and individual patient data meta-analysis
}

\author{
Leticia A Deveza, ${ }^{1}$ Sita M A Bierma-Zeinstra, ${ }^{2,3}$ Willem Evert van Spil, ${ }^{4}$ \\ Win Min Oo, ${ }^{1}$ Bruno T Saragiotto, ${ }^{5,6}$ Tuhina Neogi, ${ }^{7}$ Marienke van Middelkoop, ${ }^{3}$ \\ David J Hunter ${ }^{1}$
}

To cite: Deveza LA, BiermaZeinstra SMA, van Spil WE, et al. Efficacy of bisphosphonates in specific knee osteoarthritis subpopulations: protocol for an OA Trial Bank systematic review and individual patient data meta-analysis. BMJ Open 2018;8:e023889. doi:10.1136/ bmjopen-2018-023889

- Prepublication history and additional material for this paper are available online. To view these files, please visit the journal online (http://dx.doi org/10.1136/bmjopen-2018023889).

Received 28 April 2018 Revised 6 August 2018 Accepted 5 November 2018

Check for updates

(c) Author(s) (or their employer(s)) 2018. Re-use permitted under CC BY-NC. No commercial re-use. See rights and permissions. Published by BMJ.

For numbered affiliations see end of article.

Correspondence to

Dr Leticia A Deveza;

leticia.alle@sydney.edu.au

\section{ABSTRACT}

Introduction Randomised clinical trials to date investigating the efficacy of bisphosphonates in knee osteoarthritis $(0 \mathrm{~A})$ have found divergent results, with a recent meta-analysis finding no superiority of these drugs over placebo. Whether particular patient subgroups are more likely to benefit from this therapy than others is still unclear. We aim to investigate the effects of bisphosphonates compared with a control group (placebo, no treatment, another active treatment) on clinical and structural outcomes in specific knee OA subpopulations with possible distinct rates of subchondral bone turnover.

Methods and analysis Medline, Embase, Scopus, Web of Sciences and Cochrane Central Register of Controlled Trials will be searched from inception to February 2018. Randomised clinical trials will be eligible if they reported at least one potential treatment effect modifier at baseline: gender, menopausal status, age, body mass index, radiographic stage, knee pain severity, presence of bone marrow lesions, levels of biochemical markers of bone turnover (serum and/or urinary) and systemic bone mineral density status. Authors of original trials will be contacted to obtain individual patient data from each study. Risk of bias will be assessed using the Cochrane Collaboration's tool. The primary outcomes will include pain and radiographic joint space width loss. Studies using other MRI-based assessment of disease progression will also be eligible. Outcomes will be grouped into short-term ( $\leq 3$ months), intermediate-term ( $>3$ months; $\leq 12$ months) and long-term (>12 months). Regression models will be used, adding an interaction term for each subgroup of interest to determine possible subgroup effects. There was no source of funding for this study.

Ethics and dissemination Dissemination of our findings is planned to occur through conference presentations, publication in peer-reviewed journals and social media. No formal ethics approval is generally required as no new data collection will be undertaken.
Strengths and limitations of this study

- Particular subgroups of patients with knee osteoarthritis $(O A)$ may be more likely to benefit from bisphosphonate therapy than others. Previous clinical studies, including conventional meta-analyses, were unable to address this important issue.

- We will use individual patient data meta-analysis, which allows for more consistent identification and extraction of common individual-level characteristics, standardised outcome measures and greater statistical power to conduct subgroup analyses.

- The OA Trial Bank is an international initiative aimed to foster research on the identification of $O A$ patient subgroups for targeted treatment. A steering committee has assessed and approved this study initiative and will provide support for all procedures related to data collection, transfer and management.

- The analyses planned will depend on availability of data provided for this study.

- The variables that will be tested as potential treatment effect modifiers are possibly associated with but are not direct measures of distinct rates of subchondral bone turnover.

PROSPERO registration number CRD42018093327.

\section{INTRODUCTION}

Osteoarthritis (OA) affects more people than any other type of arthritis. ${ }^{1}$ The overall lifetime risk of suffering from knee $\mathrm{OA}$ is estimated to range from $14 \%$ to $44 \%$ for the general population. $^{23}$ However, specific subpopulations are at higher risk: around $20 \%$ to $60 \%$ of obese individuals and $34 \%$ to $56 \%$ of individuals with significant knee injury will develop knee OA. ${ }^{24}$ OA was ranked the 12 th major cause of global disability in 2016 among all other medical conditions and showed one of 
the greatest rises in years lived with disability over the last decade. ${ }^{5}$ This high and growing burden of OA threatens healthcare systems and desperately demands more efficacious treatments to prevent the structural and clinical deterioration associated with this condition.

Subchondral bone is a potential target in $\mathrm{OA}$ as there is extensive literature showing the important role of subchondral bone remodelling in OA pathogenesis. ${ }^{6-9}$ Microstructural subchondral bone changes start early in the OA process, promoting neovascularisation and growth of sensory nerves to the deepest cartilage layers, which facilitate the exchange of local factors between these tissues. ${ }^{6}$ Preclinical studies have demonstrated that bone and cartilage interact through biochemical, cellular and mechanical mechanisms, which contribute to joint pain, changes in cartilage homoeostasis and subsequent joint damage. ${ }^{9} 10$ The subchondral bone in OA knees has been characterised as having a high turnover state, resulting in relative hypomineralisation and bone weakness; however, increased subchondral bone thickness and volume have also been observed, which might result in an overall increase in subchondral bone stiffness. ${ }^{10} \mathrm{~A}$ preclinical study suggested an increase in bone resorption in the onset of OA development while increased bone formation prevails in later stages. ${ }^{11}$ Nevertheless, early subchondral bone changes in human OA joints are still less understood.

Bisphosphonates are a class of medication commonly used in the treatment of osteoporosis and other metabolic bone disorders, acting primarily on osteoclasts in active remodelling sites in the skeleton. ${ }^{12}$ Through the inhibition of osteoclast-induced bone resorption, bisphosphonates decrease bone remodelling and improve bone mineralisation and trabecular microarchitecture. ${ }^{12}$ Numerous preclinical studies have shown a protective effect of bisphosphonates on joint structure, improving subchondral bone quality and protecting the hyaline cartilage from damage, ${ }^{13} 14$ in addition to a possible reduction in synovitis. ${ }^{14} 15$ Moreover, observational clinical studies have suggested a potential benefit of bisphosphonates in reducing knee pain, ${ }^{16}$ joint space width loss, ${ }^{16}$ spinal osteophyte progression ${ }^{17}$ and rate of total knee replacement. ${ }^{18} 19$ However, a few of these studies were specifically conducted in populations with osteoporosis and it is unclear whether the subchondral bone in these individuals is more prone to beneficially react to the effect of bisphosphonates. ${ }^{171920}$ Despite these encouraging results, clinical trials to date testing the effectiveness of bisphosphonates on clinical and structural outcomes have found mixed results. ${ }^{21}$ Moreover, a recent systematic review and meta-analysis on bisphosphonates in knee OA including seven randomised, placebo-controlled trials (3013 patients) found no differences in pain, function and the proportion of patients experiencing radiographic progression. ${ }^{22}$

There is a growing body of literature describing heterogeneity in the pathogenesis and clinical and structural manifestations of OA. ${ }^{23}$ It has been suggested that increased subchondral bone remodelling may not be universal to all patients or through all stages of the disease process. ${ }^{24-27}$ As a consequence, targeting the OA population as a whole may conceal beneficial effects of bisphosphonates if only specific subgroups of patients experience benefits from the therapy. Other authors have highlighted the need to identify the 'right people at the right time' in whom bisphosphonates may be of benefit. ${ }^{24}$ Post-menopausal women with decreased subchondral bone density and increased remodelling, ${ }^{25}$ early OA stages ${ }^{26} 28$ and presence of bone marrow lesions (BMLs) ${ }^{29} 30$ were particularly highlighted as potential subpopulations likely to benefit from bisphosphonates. ${ }^{27}$ In addition, there is a marked difference in potency across different types of bisphosphonates. This variation is mainly related to the presence of a nitrogen molecule in the structure of the most contemporaneous bisphosphonate drugs, which could also be a factor affecting the magnitude of treatment effect. ${ }^{12}$

The OA Trial Bank is an initiative aimed to foster research on the identification of OA patient subgroups for targeted treatment through individual patient data (IPD) meta-analysis. ${ }^{31}{ }^{32}$ IPD meta-analysis is the gold standard for reviews aiming to synthesise the evidence of clinical studies and is a useful technique to conduct well-defined subgroup analyses as it enables more consistent identification and extraction of common individual-level characteristics, standardised outcome measures and greater statistical power to conduct subgroup analyses. ${ }^{33} 34$ Prior conventional meta-analyses on bisphosphonates in OA were unable to address this important issue of identifying subgroups of individuals who may most benefit from bisphosphonate therapy. ${ }^{21} 2235$ The steering committee of the OA Trial Bank ensures the appropriateness of the research question and methodology and a strategy is in place to contact the authors of eligible clinical trials in order to obtain original research data from each study.

The unacceptable lack of disease-modifying agents in OA combined with suggestive evidence of a role for bisphosphonates in selected patient subgroups underlies the need for methodologically sound studies to potentially identify differential effects of bisphosphonates in predefined knee OA subpopulations. Under the expertise of the OA Trial Bank, we aim to conduct a systematic review with IPD meta-analysis to perform subgroup analysis of existing clinical trials on the efficacy of bisphosphonates on clinical and structural outcomes in patients with knee OA.

\section{METHODS}

A summary of the study proposal along with the research question has been approved by the steering committee of the OA Trial Bank before the preparation of the full study protocol. We followed the Preferred Reporting Items for Systematic Reviews and Meta-Analyses-IPD (PRISMA-IPD) checklist to ensure the quality of reporting of this systematic review and IPD meta-analysis protocol. ${ }^{36}$ 


\section{Literature search}

\section{Identification of studies}

We will search the following databases: Medline, Embase, Scopus, Web of Sciences and Cochrane Central Register of Controlled Trials from inception to February 2018. Reference lists of selected studies and relevant reviews will be hand searched for additional studies and Google Scholar will be searched for additional citations associated with the final included studies. The search strategy will contain a combination of key search terms (and related terms) including three main filters: (1) population: OA and knee; (2) intervention: bisphosphonate, clodronate, tiludronate, etidronate, alendronate, zoledronic acid (or zoledronate), pamidronate, risedronate, neridronate, ibandronate, olpadronate, incadronate; (3) study design: randomised and trial. The full search strategy can be found in online supplementary appendix 1. All titles and abstracts will be imported into EndNote X7 for the screening process.

\section{Screening process}

Two independent reviewers (LAD, WMO) will screen all titles and abstracts for potentially eligible studies. In a second step, the full text of the selected studies will be assessed for eligibility by the same independent reviewers and any disagreements will be discussed and resolved with a third reviewer $(\mathrm{DJH})$ if consensus is not achieved. The results of the literature search will be summarised in a flowchart as suggested by the PRISMA guidelines. ${ }^{37}$

\section{Study selection}

All trials that fulfil the following eligibility criteria will be included in the IPD meta-analysis.

\section{Inclusion criteria}

\section{Participants}

Studies including participants with a diagnosis of symptomatic knee OA according to the American College of Rheumatology classification criteria, ${ }^{38}$ physician diagnosis or other diagnostic criteria based on clinical manifestations, with or without radiographic data.

\section{Intervention}

All bisphosphonates will be considered for the purpose of this study regardless of the route of administration (oral, intravenous or intra-articular), dosage or molecular characteristics (nitrogenous vs non-nitrogenous).

\section{Control}

Trials comparing bisphosphonate treatment with a control group (placebo, no treatment or another active treatment) will be eligible.

\section{Outcome}

Trials will be eligible if there are individual patient data available for both bisphosphonate and control groups on at least one of the following outcomes: knee pain (primary clinical outcome), irrespective of the scale used, or structural disease progression based on radiographic joint space width (JSW) (primary structural outcome) or other MRI-based assessment of disease progression, at baseline and any follow-up time points.

\section{Study design}

Only studies that are randomised clinical trials will be eligible.

Variables for subgroup analyses: All studies included in the IPD meta-analysis should have at least one of the variables that will be used as potential treatment effect modifiers assessed at baseline (described in the 'Subgroup Analyses' section below).

\section{Exclusion criteria}

Studies in which the intervention group received bisphosphonate combined with another active treatment (pharmacological or non-pharmacological) will be excluded. We will also exclude studies exclusively assessing the effect of bisphosphonates in animals or in joints other than the knee, reviews, editorials, conference abstracts with no other source of data available, and non-randomised trials. There will be no language restriction.

\section{Data acquisition, transfer and checks}

We will use the same strategy for data acquisition and transfer used in all other studies in the OA Trial Bank. ${ }^{31}{ }^{39}$ The corresponding author of the eligible trials will be contacted first by email with two subsequent email reminders, followed by telephone if needed. If the corresponding author is unavailable, the other trial authors and/or institutions listed will be contacted. On favourable response and willingness to share the data, data deliverers will be asked to sign the data delivery license agreement, which will contain items on input data, obligations, ownership of data, terms, authorship, subgroup analyses planned and dissemination of the results. All de-identified data will be transferred to a secure database at Erasmus University Medical Center, Rotterdam, the Netherlands. Data sets will be accepted in any format, provided that variables and categories are adequately labelled within the data set or with a separate codebook. To ensure quality, data from all studies will be checked for duplicates and completeness. Outliers or unusual values will be assessed by range checks. Numbers related to participants baseline characteristics and changes over time for the primary outcome measures will be compared with published papers to check for consistency. In case of differences, authors of the original studies will be contacted to clarify any discrepancies.

\section{Methodological quality assessment}

Two raters (LAD, WEvS) will independently assess the risk of bias and the quality of evidence of the included studies using the 12 items from the Cochrane Collaboration's tool. ${ }^{40}$ The following areas will be assessed: sequence generation; allocation concealment; blinding of participants, personnel and outcome assessment; incomplete outcome data; selective reporting and other sources of bias. Each item will be scored as yes (low risk 


\begin{tabular}{|c|c|}
\hline Characteristic & Description \\
\hline Gender & Male vs female \\
\hline Menopausal status & $\begin{array}{l}\text { Premenopausal vs } \\
\text { postmenopausal }\end{array}$ \\
\hline Age (years) & Continuous variable \\
\hline Body mass index* (BMI) & $\begin{array}{l}\mathrm{BMI} \geq 30 \text { (obese) vs }<30 \\
\text { (non-obese) }\end{array}$ \\
\hline $\begin{array}{l}\text { Radiographic osteoarthritis } \\
\text { (OA) stage }\end{array}$ & $\begin{array}{l}\text { Radiographic minimum joint } \\
\text { space width (continuous); } \\
\text { Kellgren Lawrence grade }\end{array}$ \\
\hline Baseline knee pain severity & Continuous variable \\
\hline Bone marrow lesion (BML) & $\begin{array}{l}\text { Presence of at least one } \\
\text { moderate to large BML vs } \\
\text { absence of presence of } \\
\text { small BML }\end{array}$ \\
\hline $\begin{array}{l}\text { Baseline levels of systemic } \\
\text { markers of bone turnover (eg, } \\
\text { CTX-I, CTX-II and NTX-I) }\end{array}$ & $\begin{array}{l}\geq \text { mean value vs }< \\
\text { mean value } †\end{array}$ \\
\hline
\end{tabular}

Systemic bone density status Either as continuous variable or presence vs absence of osteopenia/osteoporosis

*Measured in $\mathrm{kg} \mathrm{m}^{-2}$.

†Values will be standardised if the distribution is skewed.

of bias), no (high risk of bias) or unclear. A low risk of bias study will be defined as fulfilling six or more of the criteria items, as previously used in other studies in the OA Trial Bank. ${ }^{39} 41$ Any disagreements will be discussed and resolved by consensus or consulting a third reviewer (BTS).

\section{Subgroup analyses}

The characteristics that will be assessed as potential treatment effect modifiers if sufficient data are available are (table 1): (1) gender: male vs female; (2) menopausal status: premenopausal vs post-menopausal; (3) age (as continuous variable); (4) body mass index (BMI): obese $\left(\mathrm{BMI} \geq 30 \mathrm{~kg} \mathrm{~m}^{-2}\right.$ ) vs non-obese; (5) radiographic disease stage, assessed by baseline radiographic minimum JSW or Kellgren Lawrence grade, ${ }^{42}$ depending on data availability; (6) knee pain severity at baseline (as continuous variable); (7) BMLs: presence of at least one moderate or large BML on MRI vs absence or presence of small BML; (8) baseline levels of serum or urinary biochemical markers of bone turnover (eg, CTX-I, CTX-II and NTX-I): $\geq$ or $<$ mean value (values will be transformed if the distribution is skewed); and (9) systemic bone mineral density status (either as continuous variable or presence vs absence of osteopenia/osteoporosis).

\section{Data analysis and reporting}

A descriptive synthesis of each study and participants' demographic characteristics will be conducted. Publication bias will be investigated using funnel plot analysis. ${ }^{43}$ Assessment of heterogeneity across trials for the primary outcomes will be based on the $\mathrm{I}^{2}$. A complete case analysis will be carried out preferably over imputation of missing data. If a high proportion of missing data is encountered, multiple imputation will be used for potential treatment effect modifiers and covariates within each trial due to missing at random assumption before the results are pooled $^{44}$ and a sensitivity analysis will be carried out to assess any difference in results compared with the non-imputed data set.

For the analysis of the magnitude of treatment effect in the whole population, including all studies, and in each specific subgroup of interest described above, a one-step approach meta-analysis will be carried out as the primary analysis. ${ }^{45}$ This is done by appending the data from all studies after appropriate standardisation of variables and outcomes has taken place and performing the analyses in this new dataset. To assess for possible subgroup effects, interaction effects will be determined using random-effects regression models, adding an interaction term for each subgroup of interest and adjusting for a variable representing the study to account for residual confounding by study differences. As a secondary analysis, a two-stage meta-analysis approach will be used. According to this approach, subgroup analysis will be performed within each trial for all standardised outcomes. In a second step, interaction terms will be pooled for each potential predictor using random-effects models in the same way as conventional meta-analysis with aggregated data. The main comparison will be made between bisphosphonate and placebo and other comparisons will be planned according to the availability of trials and data.

Risk ratios and $95 \%$ CI will be the preferred measure of effect to be presented for dichotomous outcomes (radiographic progression, other MRI-based assessment of disease progression and progression to total knee replacement). Mean differences and 95\% CI will be presented for continuous outcomes (knee pain and physical function) if the same scale was used for outcome assessment across trials or standardised mean differences and $95 \%$ CI if different scales were used. Primary outcomes (pain and radiographic JSW) will be grouped into three main timepoints of assessment: short term ( $\leq 3$ months), intermediate term ( $>3$ months $; \leq 12$ months) and long term ( $>12$ months). Radiographic assessment of progression based on JSW will be dichotomised into progression if JSW loss $\geq 0.7 \mathrm{~mm}$ or non-progression if JSW loss $<0.7 \mathrm{~mm}$ if JSW measurements are available, which is the smallest detectable difference of this method. ${ }^{46}$ Secondary outcomes will include physical function, progression to total knee replacement and structural progression based on other OA-related features on MRI and radiograph such as Kellgren Lawrence grade (KLG) ${ }^{42}$ and size or volume of BMLs, if available. The incidence of adverse events and serious adverse events will be compared between the intervention and control groups using IPD from all studies. We will use intention-to-treat analysis preferably over per-protocol or as-treated analysis. The results will be interpreted taking into account the number of trials 
and participants included in the analyses and the methodological quality of the trials.

To determine the influence of bisphosphonate type on pooled treatment effects, analyses for the primary outcomes will be stratified by route of administration (ie, intravenous vs oral vs. intra-articular) and molecular characteristics (nitrogeneous vs non-nitrogenous) using data from all trials included in the meta-analysis.

\section{Patient and public involvement}

There are two members of the Arthritis Research UK OA Research Users Group who are representatives of patient and public involvement in the Steering Committee of the OA Trial Bank. The Steering Committee assesses all study proposals before the preparation of the protocol and approves and agrees on the study's design and methods, subgroups proposed and statistical analysis.

\section{ETHICS AND DISSEMINATION}

No formal ethics approval is generally required as no new data collection will be undertaken. If ethics approval is required in order to perform new analyses in any individual eligible study, approval will be requested and obtained before conducting any additional analysis. A data delivery license agreement will be provided and signed by one of the authors of each of the original trials providing data for this study. Dissemination of our findings is planned to occur through presentations at international conferences, publication in peer-reviewed journals and social media such as Twitter.

\section{Author affiliations}

${ }^{1}$ Rheumatology Department, Royal North Shore Hospital and Institute of Bone and Joint Research, Kolling Institute, University of Sydney, Sydney, New South Wales, Australia

${ }^{2}$ Department of Orthopedic Surgery, Erasmus MC, University Medical Centre Rotterdam, Rotterdam, The Netherlands

${ }^{3}$ Department of General Practice, Erasmus University Medical Centre, Rotterdam, Netherlands

${ }^{4}$ Department of Rheumatology and Clinical Immunology, University Medical Center Utrecht, Utrecht, The Netherlands

${ }^{5}$ School of Public Health, Sydney Medical School, The University of Sydney, Sydney, New South Wales, Australia

${ }^{6}$ Centre for Pain, Health and Lifestyle, Sydney, New South Wales, Australia

${ }^{7}$ Clinical Epidemiology Research and Training Unit, Boston University School of

Medicine, Boston, Massachusetts, USA

Contributors LAD, SMAB-Z, WEvS and DJH contributed to the study conception. LAD, SMAB-Z, WEvS, WMO, BTS, TN, MvM and DJH contributed to the study design. $\angle A D$ developed the search strategy and drafted the first version of the manuscript. LAD, SMAB-Z, WEvS, WMO, BTS, TN, MvM and DJH revised the protocol critically for important intellectual content, read and approved the final version of the protocol and agree to be accountable for all aspects of the work in ensuring that questions related to the accuracy or integrity of any part of the work are appropriately investigated and resolved. The study proposal has been peer reviewed and approved by the OA Trial Bank Steering Committee.

Funding The authors have not declared a specific grant for this research from any funding agency in the public, commercial or not-for-profit sectors.

Competing interests Professor David Hunter reports personal fees from consulting fees from Merck Serono, Flexion and Tissuegene, outside the submitted work. Prof. Bierma-Zeinstra reports grants from the European Union, The Netherlands Organisation for Health Research and Development, Dutch Arthritis Foundation,
CZ, Nuts Ohra, Stichting Coolsingel, personal fees from Infirst Healthcare and from Osteoarthritis and Cartilage, outside the submitted work. All other authors have nothing to disclose.

\section{Patient consent Not required.}

Provenance and peer review Not commissioned; externally peer reviewed.

Open access This is an open access article distributed in accordance with the Creative Commons Attribution Non Commercial (CC BY-NC 4.0) license, which permits others to distribute, remix, adapt, build upon this work non-commercially, and license their derivative works on different terms, provided the original work is properly cited, appropriate credit is given, any changes made indicated, and the use is non-commercial. See: http://creativecommons.org/licenses/by-nc/4.0/.

\section{REFERENCES}

1. Johnson VL, Hunter DJ. The epidemiology of osteoarthritis. Best Pract Res Clin Rheumatol 2014;28:5-15.

2. Losina E, Weinstein AM, Reichmann WM, et al. Lifetime risk and age at diagnosis of symptomatic knee osteoarthritis in the US. Arthritis Care Res 2013;65:703-11.

3. Murphy L, Schwartz TA, Helmick CG, et al. Lifetime risk of symptomatic knee osteoarthritis. Arthritis Rheum 2008;59:1207-13.

4. Suter LG, Smith SR, Katz JN, et al. Projecting lifetime risk of symptomatic knee osteoarthritis and total knee replacement in individuals sustaining a complete anterior cruciate ligament tear in early adulthood. Arthritis Care Res 2017;69:201-8.

5. Disease GBD, Injury I, Prevalence C. Global, et al. Global, regional, and national incidence, prevalence, and years lived with disability for 328 diseases and injuries for 195 countries, 1990-2016: a systematic analysis for the global burden of disease study 2016. Lancet 2017;390:1211-59.

6. Funck-Brentano T, Cohen-Solal M. Subchondral bone and osteoarthritis. Curr Opin Rheumatol 2015;27:420-6.

7. Mahjoub M, Berenbaum F, Houard X. Why subchondral bone in osteoarthritis? The importance of the cartilage bone interface in osteoarthritis. Osteoporos Int 2012;23:841-6.

8. Yu D, Xu J, Liu F, et al. Subchondral bone changes and the impacts on joint pain and articular cartilage degeneration in osteoarthritis. Clin Exp Rheumatol 2016;34:929-34.

9. Karsdal MA, Bay-Jensen AC, Lories RJ, et al. The coupling of bone and cartilage turnover in osteoarthritis: opportunities for bone antiresorptives and anabolics as potential treatments? Ann Rheum Dis 2014; 73:336-48.

10. Burr DB, Gallant MA. Bone remodelling in osteoarthritis. Nat Rev Rheumatol 2012;8:665-73.

11. Hayami T, Pickarski M, Zhuo Y, et al. Characterization of articular cartilage and subchondral bone changes in the rat anterior cruciate ligament transection and meniscectomized models of osteoarthritis. Bone 2006;38:234-43.

12. Papapoulos SE. Bisphosphonates: how do they work? Best Pract Res Clin Endocrinol Metab 2008;22:831-47.

13. Funck-Brentano T, Lin H, Hay E, et al. Targeting bone alleviates osteoarthritis in osteopenic mice and modulates cartilage catabolism. PLoS One 2012;7:e33543.

14. Siebelt M, Waarsing JH, Groen HC, et al. Inhibited osteoclastic bone resorption through alendronate treatment in rats reduces severe Osteoarthritis progression. Bone 2014;66:163-70.

15. Moreau M, Rialland P, Pelletier JP, et al. Tiludronate treatment improves structural changes and symptoms of Osteoarthritis in the canine anterior cruciate ligament model. Arthritis Res Ther 2011;13:R98.

16. Laslett LL, Kingsbury SR, Hensor EM, et al. Effect of bisphosphonate use in patients with symptomatic and radiographic knee osteoarthritis: data from the Osteoarthritis Initiative. Ann Rheum Dis 2014;73:824-30.

17. Neogi T, Nevitt MC, Ensrud KE, et al. The effect of alendronate on progression of spinal osteophytes and disc-space narrowing. Ann Rheum Dis 2008;67:1427-30.

18. Neogi T, Li S, Peloquin C, et al. Effect of bisphosphonates on knee replacement surgery. Ann Rheum Dis 2018;77:92-7.

19. Fu SH, Wang CY, Yang RS, et al. Bisphosphonate Use and the Risk of Undergoing total knee arthroplasty in Osteoporotic patients with Osteoarthritis: a nationwide cohort study in Taiwan. J Bone Joint Surg Am 2017;99:938-46.

20. Lems WF. Bisphosphonates: a therapeutic option for knee osteoarthritis? Ann Rheum Dis 2018;77:1247-8.

21. Xing RL, Zhao LR, Wang PM. Bisphosphonates therapy for osteoarthritis: a meta-analysis of randomized controlled trials. Springerplus 2016;5:5. 
22. Vaysbrot EE, Osani MC, Musetti MC, et al. Are bisphosphonates efficacious in knee osteoarthritis? A meta-analysis of randomized controlled trials. Osteoarthritis Cartilage 2018;26:154-64.

23. Deveza LA, Loeser RF. Is osteoarthritis one disease or a collection of many? Rheumatology 2018;57:iv34-iv42.

24. Walsh DA, Chapman V. Bisphosphonates for osteoarthritis. Arthritis Res Ther 2011;13:128.

25. Roman-Blas JA, Castañeda S, Largo R, et al. An OA phenotype may obtain major benefit from bone-acting agents. Semin Arthritis Rheum 2014;43:421-8

26. Saag KG. Bisphosphonates for osteoarthritis prevention: "Holy Grail" or not? Ann Rheum Dis 2008;67:1358-9.

27. Herrero-Beaumont G, Roman-Blas JA. Osteoarthritis: Osteoporotic OA: a reasonable target for bone-acting agents. Nat Rev Rheumatol 2013;9:448-50.

28. Strassle BW, Mark L, Leventhal L, et al. Inhibition of osteoclasts prevents cartilage loss and pain in a rat model of degenerative joint disease. Osteoarthritis Cartilage 2010;18:1319-28.

29. Varenna M, Zucchi F, Failoni S, et al. Intravenous neridronate in the treatment of acute painful knee osteoarthritis: a randomized controlled study. Rheumatology 2015;54:1826-32.

30. Laslett LL, Doré DA, Quinn SJ, et al. Zoledronic acid reduces knee pain and bone marrow lesions over 1 year: a randomised controlled trial. Ann Rheum Dis 2012;71:1322-8.

31. van Middelkoop M, Dziedzic KS, Doherty M, et al. Individual patient data meta-analysis of trials investigating the effectiveness of intra-articular glucocorticoid injections in patients with knee or hip osteoarthritis: an OA trial bank protocol for a systematic review. Syst Rev 2013;2:54.

32. The OA Trial Bank. Inidividual patient data meta-analysis in osteoarthritis research. http://www.oatrialbank.com/home (Accessed 8 Feb 2018).

33. Stewart LA, Tierney JF, Clarke M. Chapter 19: reviews of individual patient data. In: Higgins JPT, Green S, eds. Cochrane handbook for systematic reviews of interventions Version 5.1.0 (updated March 2011): The Cochrane Collaboration, 2011.

34. Riley RD, Lambert PC, Abo-Zaid G. Meta-analysis of individual participant data: rationale, conduct, and reporting. $B M J$ 2010;340:c221.
35. Davis AJ, Smith TO, Hing CB, et al. Are bisphosphonates effective in the treatment of osteoarthritis pain? A meta-analysis and systematic review. PLOS One 2013;8:e72714.

36. Stewart LA, Clarke M, Rovers $M$, et al. Preferred Reporting Items for Systematic Review and Meta-Analyses of individual participant data: the PRISMA-IPD Statement. JAMA 2015;313:1657-65.

37. Moher $D$, Liberati $A$, Tetzlaff $J$, et al. Preferred reporting items for systematic reviews and meta-analyses: the PRISMA statement. BMJ 2009;339:b2535.

38. Altman R, Asch E, Bloch D, et al. Development of criteria for the classification and reporting of osteoarthritis. Classification of osteoarthritis of the knee. Diagnostic and therapeutic criteria committee of the American Rheumatism Association. Arthritis Rheum 1986;29:1039-49.

39. Runhaar J, Rozendaal RM, van Middelkoop M, et al. Subgroup analyses of the effectiveness of oral glucosamine for knee and hip osteoarthritis: a systematic review and individual patient data metaanalysis from the OA trial bank. Ann Rheum Dis 2017;76:1862-9.

40. Higgins J, Green S. In: Green S, Higgins J, Cochrane handbook for systematic review of interventions: Julian Higgins, 2011.

41. van Middelkoop M, Arden NK, Atchia I, et al. The OA Trial Bank: meta-analysis of individual patient data from knee and hip osteoarthritis trials show that patients with severe pain exhibit greater benefit from intra-articular glucocorticoids. Osteoarthritis Cartilage 2016;24:1143-52.

42. Kellgren JH, Lawrence JS. Radiological assessment of osteoarthrosis. Ann Rheum Dis 1957;16:494-502.

43. Guyatt GH, Oxman AD, Montori V, et al. GRADE guidelines: 5 . Rating the quality of evidence-publication bias. J Clin Epidemiol 2011;64:1277-82.

44. Koopman L, van der Heijden GJ, Grobbee DE, et al. Comparison of methods of handling missing data in individual patient data metaanalyses: an empirical example on antibiotics in children with acute otitis media. Am J Epidemiol 2008;167:540-5.

45. Debray TP, Moons KG, van Valkenhoef G, et al. Get real in individual participant data (IPD) meta-analysis: a review of the methodology. Res Synth Methods 2015;6:293-309.

46. Ornetti P, Brandt K, Hellio-Le Graverand MP, et al. OARSIOMERACT definition of relevant radiological progression in hip/knee osteoarthritis. Osteoarthritis Cartilage 2009;17:856-63. 